\section{Transformationslösungen zur Hämoglobinbestimmung}

\author{
A. M. Gressner ${ }^{1}$ und O. A. Gressner ${ }^{2}$ \\ ${ }^{1}$ Labor Dr. Wisplinghoff Berlin, Berlin, Deutschland \\ ${ }^{2}$ Labor Dr. Wisplinghoff Köln, Köln, Deutschland
}

Synonym(e) Umwandlungslösungen für Hämoglobinbestimmung; Drabkin-Lösung; van Kampen-Zijlstra-Lösung

Englischer Begriff transforming solution/reagent for hemoglobin measurement

Definition Chemische Umwandlung (Transformation) von Hämoglobin in die oxidierte (3-wertiges Eisen) Form des Hämiglobins mit nachfolgender Überführung in stabiles, photometrierbares Hämiglobincyanid (s. a. > Drabkin-Lösung).

Beschreibung Hämoglobin wird in einer zweistufigen chemischen „Transformation“ zunächst (I) durch Kaliumhexacyanoferrat zum dreiwertigen $\left(\mathrm{Fe}^{3+}\right)$ Hämiglobin ( $\triangleright$ Methämoglobin) oxidiert, um anschließend (II) durch Kaliumcyanid in stabiles Hämiglobincyanid (Cyanhämiglobin) überführt zu werden. Die Intensität der sich entwickelnden, stabilen, bräunlichen Farbe wird nach Abschluss der Farbentwicklung bei 546 nm photometriert gemessen ( $\triangleright$ Hämoglobin). Dabei kann die
Quantifizierung des Hämiglobins grundsätzlich über einen Standard oder über den molaren Extinktionskoeffizienten des Hämiglobincyanids erfolgen. Hämoglobin, Oxyhämoglobin, Kohlenmonoxidhämoglobin (Carboxyhämoglobin), Methämoglobin (Hämiglobin) werden quantitativ in Hämiglobincyanid überführt, nicht jedoch $\triangleright$ Sulfhämoglobin.

Im Wesentlichen sind zwei leicht verschiedene, in Farbentwicklungszeit und Farbstabilität unterschiedliche Transformationslösungen in Gebrauch:

- Drabkin-Lösung (Kaliumhexacyanoferrat, Kaliumcyanid, Natriumbicarbonat, pH 8,6): Farbentwicklung 10-20 min, Stabilität mehrere Tage.

- Van-Kampen- und Zijlstra-Lösung (Kaliumhexacyanoferrat, Kaliumcyanid, Kaliumhydrogenphosphat, pH 7,2): Farbentwicklung 4 Minuten, Stabilität 24 Stunden. Wegen der Schnelligkeit der Farbentwicklung ist diese Methode (oder ggf. weitere Modifikationen) für mechanisierte Analysensysteme von Vorteil.

\section{Literatur}

Greiling H, Gressner AM (1995) Lehrbuch der Klinischen Chemie und Pathobiochemie, 3. Aufl. F K Schattauer Verlag, Stuttgart

Hallmann L (1980) Klinische Chemie und Mikroskopie, 11. Aufl. Georg Thieme Verlag, Stuttgart 